\title{
Multi-Layered Polyamide/Collagen Scaffolds with Topical Sustained Release of N-Acetylcysteine for Promoting Wound Healing
}

This article was published in the following Dove Press journal: International Journal of Nanomedicine

\author{
Jinfei Hou ${ }^{1,2, *}$ \\ Lifeng Chen ${ }^{1,2, *}$ \\ Muran Zhou ${ }^{1,2}$ \\ Jialun $\mathrm{Li}$ iD ${ }^{1,2}$ \\ Jian Liu ${ }^{1,2}$ \\ Huimin Fang ${ }^{1,2}$ \\ Yuyang Zeng ${ }^{1,2}$ \\ Jiaming Sun ${ }^{1,2}$ \\ Zhenxing Wang ${ }^{1,2}$ \\ 'Department of Plastic Surgery, Union \\ Hospital, Tongii Medical College, \\ Huazhong University of Science and \\ Technology, Wuhan 430022, People's \\ Republic of China; ${ }^{2}$ Wuhan Clinical \\ Research Center for Superficial Organ \\ Reconstruction, Wuhan 430022, China \\ *These authors contributed equally to \\ this work
}

Background: Impaired wound healing might be associated with many issues, especially overactive of reactive oxygen species (ROS), deficiency of blood vessels and immature of epidermis. $\mathrm{N}$-acetylcysteine (NAC), as an antioxidant, could solve these problems by inhibiting overreactive of ROS, promoting revascularization and accelerating re-epithelialization. How to deliver NAC in situ with a controllable releasing speed still remain a challenge.

Materials and Methods: In this study, we combined collagen (Col) with $\mathrm{N}$-acetylcysteine to perform the characteristics of sustained release and chemically crosslinked $\mathrm{Col} / \mathrm{NAC}$ composite with polyamide (PA) nanofibers to enhance the mechanical property of collagen and fabricated this multi-layered scaffold (PA-Col/NAC scaffold). The physical properties of the scaffolds such as surface characteristics, water absorption and tensile modulus were tested. Meanwhile, the ability to promote wound healing in vitro and in vivo were investigated.

Results: These scaffolds were porous and performed great water absorption. The PA-Col /NAC scaffold could sustainably release NAC for at least 14 days. After cell implantation, PA$\mathrm{Col} / \mathrm{NAC}$ scaffold showed better cell proliferation and cell migration than the other groups. In vivo, PA-Col/NAC scaffolds could promote wound healing best among all the groups.

Conclusion: The multi-layered scaffolds could obviously accelerate the process of wound healing and exert better and prolonged effects.

Keywords: polyamide, sustained release, $\mathrm{N}$-acetylcysteine, wound healing, multi-layered structure

\section{Introduction}

Impaired skin regeneration is a common outcome in patients with diabetic ulcers, burns and pressure ulcers. ${ }^{1}$ Among the clinical classical wound treatments, topical therapies such as covering the wound with dressings has been widely used. Over the past decades, many composite synthetic polymers and biological materials have been used to fabricate wound dressing agents. ${ }^{2}$

The wound healing process is extremely complex and is regulated by interactions among several factors, all of which promote the reconstruction of a skin barrier. ${ }^{3}$ The reconstruction process is impaired by events such as hematoma, overreactive inflammation (reactive oxygen species) and limited angiogenesis. ${ }^{4}$ $\mathrm{N}$-acetylcysteine (NAC) is an antioxidant approved by the Food and Drug Administration for clinical use in the treatment of oxidative stress-related diseases such as diabetic ulcers. ${ }^{5,6}$ Previous studies have reported that NAC promotes wound healing by alleviating the adverse effects of ROS. ${ }^{7}$ NAC accelerates this process by
Correspondence: Jiaming Sun; Zhenxing Wang

Email sunjm1592@sina.com;

benjamin.wzx@163.com
International Journal of Nanomedicine 2020:I5 |349-|36|

1349 
improving perfusion and angiogenesis at ischemic areas. ${ }^{8}$ NAC has also been reported to facilitate maturation of epidermis through the promotion of MMP-1 expression via the PKC/Stat3 signaling pathway. ${ }^{9}$ NAC has been widely used to treat lung diseases such as chronic obstructive pulmonary disease and hepatic damage, however, rarely is it applied for wound healing. ${ }^{10,11}$ Thus, whether topical administration of NAC promotes the wound healing process has not been sufficiently studied. For this reason, further studies are required to explore its safety and mechanisms. ${ }^{12}$ Besides, chronic wounds that take long to heal may require repeated administration of NAC. This repeated administration might be feasible, but not always the best treatment strategy. ${ }^{13}$ So, the best strategy is to develop a new kind of wound dressing with the ability to achieve the long-term drug release. This strategy could decrease the frequency of changing the wound dressing and relieve the pain of patients. ${ }^{14}$

To study the topical effects of NAC and its sustained release characteristics, collagen, which has been widely used for tissue engineering and soft tissue repair, was used as the drug carrier. ${ }^{15}$ Collagen has been associated with low antigenicity and excellent biocompatibility with most endogenous tissues. Therefore, collagen-based wound dressings are used for chronic wound and ulcers treatment. $^{16-18}$ Collagen can be crosslinked with drugs to create a sustained release formulation. This ensures that there is sufficient concentration of the drug on the wound during the long healing process. ${ }^{19}$ Unfortunately, collagen is mechanically weak and prone to tear, hence, cannot be applied to wounds in some parts of the body such as joints. Although collagen can be cross-linked to enhance its mechanical properties, it is still sub-optimal to be used as an abrasion-resistant wound dressing. ${ }^{20}$

Consequently, in order to overcome the limitations associated with collagen, polyamide (PA) was chosen in this study to strengthen the mechanical properties of drugloaded collagen. Polyamide was chosen because of the following reasons: i) Polyamide, is a biocompatible material, which is widely used in the clinical treatment of wounds. ii) Polyamide is the most common biomaterial used as a suture in cutaneous surgery. ${ }^{21}$ Previous studies have reported the use of polyamides in the preparation of membranes by electrospinning technique for wound healing application. ${ }^{22,23}$

In this study, collagen was crosslinked with NAC to achieve sustained release of NAC and then fabricated into triple-layered PA-Col/NAC scaffolds, which consisted of
PA nanofibers at the core and the collagen on both sides. This multi-layered structure made use of the mechanical properties of PA nanofibers and improved the stability of the entire scaffolds. Electron microscopy, FTIR spectroscopy and Raman spectroscopy were used to observe the surface structure and characteristics of the scaffolds. The physical properties such as tensile modulus and water absorption were also measured. The migration and proliferation of NIH 3T3 cells were tested after the cells were implanted on the scaffolds to examine the effects of the scaffolds on the activity of fibroblasts. The scaffolds were implanted in Sprague Dawley (SD) rats with a dorsal wound to evaluate their properties in the promotion of wound healing. The full thickness of the skin at the wound area was excised and processed with $\mathrm{HE}$, Masson and CD31 staining to investigate wound closure after 14 days of implantation.

\section{Materials and Methods}

\section{Preparation of the PA-Col/NAC Scaffolds}

Figure 1 shows the general preparation procedure of the PACol/NAC scaffold. Firstly, polyamide 6,6 (Sigma-Aldrich, America) were dissolved in a mixture of dichloromethane (DCM, Fisher Chemical, America) and N, N-dimethylformamide (DMF, Fisher Chemical, America) in a 4:1 volume ratio and used to prepare a $15 \% \mathrm{PA}$ solution. Using the PA solution, the PA nanofibers were electrospun (voltage: $18 \mathrm{kV}$, needle: 20 gauge, speed: $1 \mathrm{~mL} / \mathrm{h}$ ). This was followed by repeated washing (5-6 times) of the PA nanofibers in 75\% alcohol (Fisher Chemical, America), deionized water and phosphate buffer saline (PBS, Fisher Chemical, America) to remove all solvent residues. Collagen was dissolved in 0.05 $\mathrm{mmol} / \mathrm{l}$ acetic acid to prepare a $14 \%$ collagen solution. The collagen solution was added into a petri dish with a diameter of $60 \mathrm{~mm}$ and a height of 2-3 mm. The processed PA nanofibers ( $3 \mathrm{~cm} * 3 \mathrm{~cm}$ square) were put on this solution and the petri dish was filled with a similar height of the collagen solution. After lyophilization $\left(40 \mathrm{kPa},-20^{\circ} \mathrm{C}\right)$, the PA-Col scaffolds were obtained and placed in NAC-EDC/NHS solution, which indicates a mixture of $50 \mathrm{mmol} / \mathrm{L}$ 1-(3-Dimethylaminopropyl)-3-ethylcarbodiimide (EDC, Sigma-Aldrich, America) and $13.5 \mathrm{mmol} / \mathrm{L} \mathrm{N}-H y d r o x y s u c c i n i m i d e ~(N H S$, Sigma-Aldrich, America) in $75 \%$ alcohol solution containing $\mathrm{NAC}$ at a concentration of $0.5 \mathrm{~g} / \mathrm{L}$. Crosslinking was then performed at room temperature for $24 \mathrm{hrs}$. Finally, the materials were rinsed and sterilized to obtain PA-Col/NAC scaffolds. 


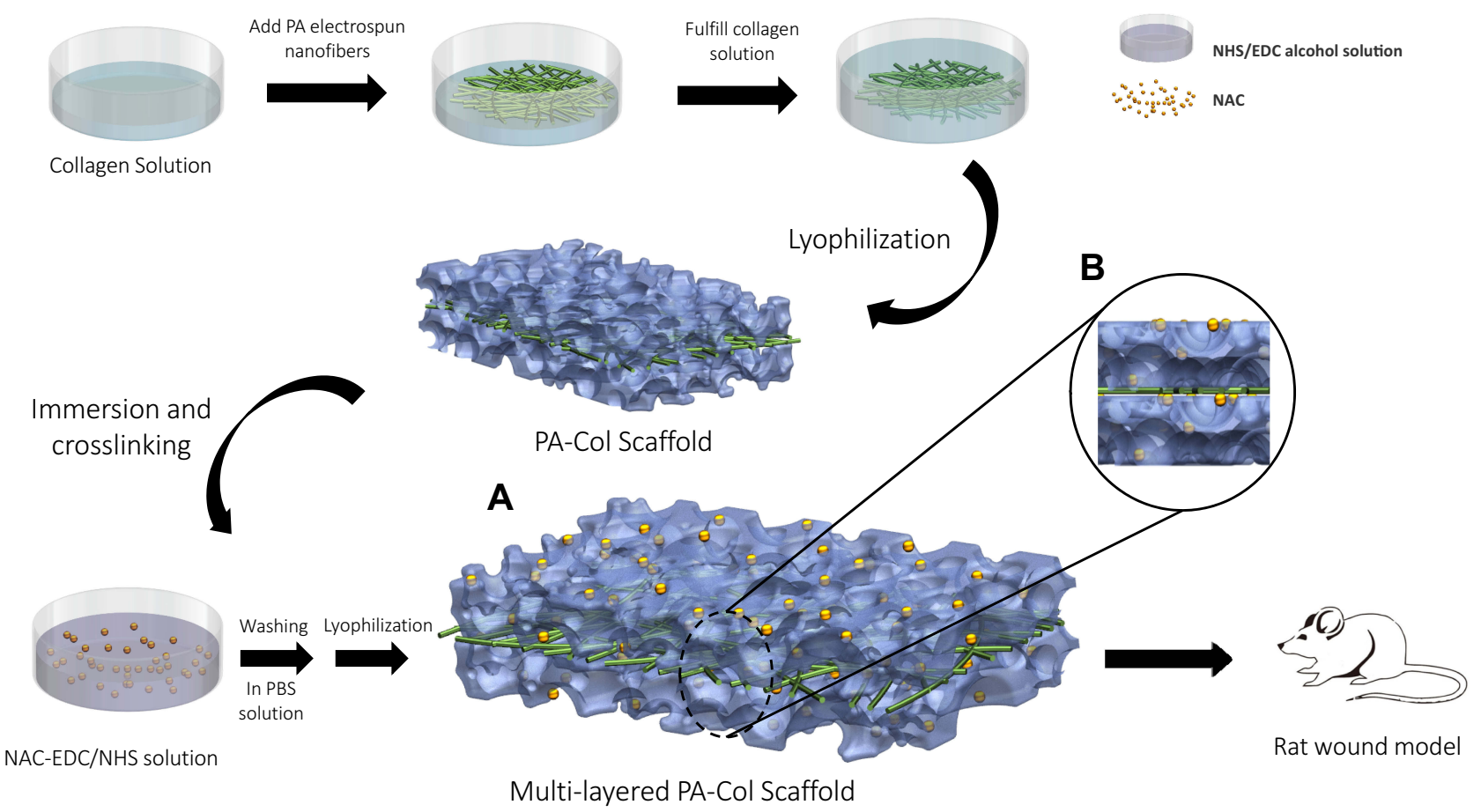

Figure I Schematic demonstrated the fabrication procedure for this multi-layered scaffold.

Notes: The culture dish was added collagen solution and PA nanofibers were put onto it. After filled with the same height of collagen solution, the composite was under lyophilization. Then this PA-Col scaffold was immersed and crosslinked in NAC-EDC/NHS solution. Subsequently, this scaffold was washed in PBS and freeze-drying, the fabrication of PA-Col/NAC was completed and applied on the rat wound model. (A) Illustration of the view consisted of the core (PA nanofibers) and the layers on two sides (Col crosslinked with NAC). (B) Multi-layered structure could be observed.

\section{Morphology of the PA-Col/NAC Scaffold}

Scanning electron microscopy (JSM-IT300, JEOL, Japan) set at an accelerating voltage of $10 \mathrm{kV}$ was used to observe the microstructure of the scaffolds. The surface of the scaffolds was sprayed with platinum using a sputter coater prior to examination. ATR-FTIR spectroscopy and Raman spectroscopy in VERTEX 70 (Bruker, German) and LabRAM HR800 (HORIBA JobinYvon, French) were used to record the surface characteristics of the scaffolds. FTIR measurements were carried out at a resolution of $4 \mathrm{~cm}^{-1}$ in the frequency range of $4000-500 \mathrm{~cm}^{-1}$ and The Raman spectrum was measured with a scanning range of $50-4000 \mathrm{~cm}^{-1}$. The excitation source was a diode laser with a wavelength of 532 $\mathrm{nm}$ adjusted to a power of $30 \mathrm{~mW}$. All the data were analyzed by using the software Origin.

\section{Water Absorption of the PA-Col/NAC Scaffold}

The water absorption property in vitro was examined. To mimic the areas around the wound and for convenient observations, $0.5 \mathrm{mg} / \mathrm{L}$ alkannin PBS solution was prepared and added to the scaffolds. To study the water absorption capacity of the scaffolds, the following formula was used:

$$
\text { Water absorption }(\%)=\frac{M s-M i}{M i}
$$

where, "Mi" represents the initial weight of the samples. "Ms" represents the weight of samples after being immersed in the PBS. ${ }^{24}$

\section{Mechanical Properties of the PA-Col/ NAC Scaffold}

All-electric dynamic test instrument (Instron, British) was used to measure the mechanical properties of the scaffolds. Tensile tests were performed according to the specifications of ASTM D638. The load cell and extension speeds were $100 \mathrm{~N}$ and $5 \mathrm{~mm} / \mathrm{min}$, respectively. The OriginPro Software (OriginLab, America) was used to calculate Young's modulus and plot the stress-strain curve.

\section{$\mathrm{NIH} 3 \mathrm{~T} 3$ in vitro Culture}

NIH 3T3 fibroblasts (ATCC, USA) were used to perform the cytological experiments. Briefly, the cells were cultured at $37^{\circ} \mathrm{C}$ in an atmosphere with $95 \%$ air $/ 5 \% \mathrm{CO} 2$ using Dulbecco's modified Eagle's medium (DMEM, Thermo Fisher Scientific, America) containing 10\% fetal bovine serum (FBS, Thermo Fisher Scientific, America) 
and $1 \%$ gentamycin/streptomycin (Thermo Fisher Scientific, America). ${ }^{25}$

\section{In vitro Cell Viability of the PA-Col/NAC Scaffolds}

NIH 3T3 cells were cultured on different samples to test the in vitro cell viability. The scaffolds were sterilized, cut into $5 \mathrm{~mm} * 5 \mathrm{~mm}$ slides and placed in 24-well plates. The NIH 3 T3 cells were prepared at a density of $1.0 \times 10^{4}$ cells per $\mathrm{mL}$ and $100 \mu \mathrm{L}$ of the cell suspension was added to each well. The fluid in each well was removed at 1, 3, 5 and 7 day. Then a mixture of $100 \mu \mathrm{L}$ complete medium and $10 \mu \mathrm{L} \mathrm{CCK}-8$ was added to each well and incubated for $4 \mathrm{hrs}$ at $37{ }^{\circ} \mathrm{C}$. The supernatant was then transferred into each well of a 96-well plate and absorbance was measured at $450 \mathrm{~nm}$ using an absorbance microplate reader. (BioTek ELx800, USA).

\section{Cell Adhesion and Compatibility of the PA-Col/NAC Scaffolds}

NIH 3 T3 cells were implanted on the slices made from the scaffolds and cultured for 1, 3 and 7 days to examine the cell adhesion and compatibility of the scaffolds. The NIH 3T3 fibroblasts were stained with fluorescein diacetate/ propidium iodide (FDA/PI) (Sigma-Aldrich, America) and then observed using a confocal laser microscope (Leica Microsystems, Germany).

\section{Cell Migration of the PA-Col/NAC Scaffolds}

NIH 3T3 fibroblasts were seeded in 24-well plates at a density of $0.5 \times 10^{6}$ cells/well and cultured until they reached a $90 \%$ confluence. Next, the migration of cells on the scaffold was evaluated. The fluid in each well was removed and replaced with a different extract liquor (a complete medium in which the scaffolds were immersed for $24 \mathrm{hrs}$ ). Scratches were made on the cell surface layers using a pipette tip. The cell images in each well were captured using an optical microscope (Olympus CKX41, Japan) at time 0, 8, 16 and $24 \mathrm{hrs}$ during the cell migration experiment.

\section{Drug Delivery Release of the PA-Col/ NAC Scaffold}

HPLC was used to test the drug release profile of the scaffolds and the absorbance of the solution at different timepoints ( $1 \mathrm{hr}, 2 \mathrm{hrs}, 4 \mathrm{hrs}, 8 \mathrm{hrs}, 24 \mathrm{hrs}, 48 \mathrm{hrs}, 7$ days and 14 days) was measured. At each time-point, $2 \mathrm{~mL}$ of the solution was taken from each well and added into a bottle filled with phosphate buffered solution (PBS) and scaffolds. Each well was replaced with $2 \mathrm{~mL}$ of a fresh PBS solution.

The concentration of drug was detected via absolute assessment with HPLC. Briefly, we measured the standard concentration and confirm the characteristic peak around 5 mins. (Figure below) Then we calculated the areas of characteristic peak and established simple unary linear regression between the standard concentration and peak area. According to this unary function, we calculated the concentration of NAC at different time points. The Origin software was used to plot the drug release curve for each scaffold.

\section{In vivo Implantation of the PA-Col/NAC Scaffold}

All procedures were performed in accordance with the guidelines approved by the Animal Ethical Committee of Huazhong University of Science and Technology. Eight adult SD rats (male, age: $8-10$ weeks; weight: $180-220 \mathrm{~g}$ ) were used for this in vivo implantation tests. All operations were performed by the same surgeon in a laminar clean bench under anesthesia ( $2 \%$ isoflurane inhalation). The sterilization of the scaffolds was done according to the ISO 11137 standard requirements. The hair was shaved, and the skin was disinfected and then three equal ovals (major axis $=2 \mathrm{~cm}$, minor axis $=1 \mathrm{~cm})^{26}$ full-thickness skin excisions were made on the dorsal skin via the wound model producer fabricated by 3D printing. Subsequently, the wound area was treated with different scaffolds and gross images were recorded at different time-points.

\section{Histological Analysis}

The animals were sacrificed 14 days after implantation by $\mathrm{CO}_{2}$ asphyxiation. The wound together with the surrounding skin was excised and fixed in $10 \%$ formaldehyde. The tissues were stained with Hematoxylin, Eosin and Masson's Trichrome (Sigma-Aldrich, America) to examine the epidermis, collagen and formation of new tissues. The new vessels formed during the wound healing were evaluated by CD31 staining (Sigma-Aldrich, America). ${ }^{27}$ The samples on the rats performed experiment were sectioned along the longest axis. Because the shapes of samples were not regular, two or three sections per specimen were evaluated. Ten sections were randomly selected and calculated for further histological analysis. 


\section{Statistical Analyses}

Statistical analysis was performed using GraphPad Software (USA). Data are shown as the mean \pm standard deviation. Parametric Student's $t$-test and Analysis of Variance (ANOVA) with a post hoc test by Tukey's method were used to determine the significance levels. The ${ }^{* * *}$ and $* * *$ refer to the P-value of $<0.05,<0.01$ and $<0.001$, respectively.

\section{Results}

\section{Physical Characterization of the Scaffolds}

Figure 2A and B shows the porous structure of the PA-Col NAC scaffold. The multi-layered structure, "collagen-PA nanofibers-collagen" composite, is shown in Figure $2 \mathrm{C}$ and D. So, it could be inferred that the surface structure of PACol/NAC scaffold was porous, and the spatial structure of collagen and PA nanofibers accumulated layer by layer. The ATR-FTIR spectrum and Raman spectrum analysis results of the PA-Col/NAC scaffolds are shown in Figure 2E and F. It can be observed that PA had a characteristic C-H peak at $960 \mathrm{~cm}-1$ and $930 \mathrm{~cm}-1 .{ }^{28} \mathrm{PA}-\mathrm{Col}$ and PA-Col/NAC exhibited the $>\mathrm{NH}$ and $-\mathrm{OH}$ peaks at $3300 \mathrm{~cm}-1$, amide I, amide II and amide III peaks at $1657 \mathrm{~cm}-1,1528 \mathrm{~cm}-1$ and $1300 \mathrm{~cm}-1$, which were ascribed to the presence of collagen in the scaffolds. ${ }^{29,30}$ In the PA, the amide peaks were obtained at the wavenumber mentioned previously. The FTIR spectrum revealed the NAC peaks as shown in Figure 2F. The PA-Col /NAC scaffold exhibited characteristic $\mathrm{V}(\mathrm{CH} 3)$ (symmetric) and the $\mathrm{V}(\mathrm{CH} 3)$ (asymmetric) peak at $2940 \mathrm{~cm}-1 .{ }^{31}$

Figure $2 \mathrm{G}$ shows the tensile stress-strain curve of different groups under the tensile loading. The three groups reached the ultimate tensile stress at the same ultimate strain of about $16.42 \% \pm 0.41 \%$. Before the ultimate tensile stress, the three curves were linear. The Young's modulus of PA, PA-Col and PA-Col/NAC group was calculated as follows: $20.34 \pm 2.22 \mathrm{MPa}, 8.44 \pm 1.20 \mathrm{MPa}$ and $7.88 \pm 1.90 \mathrm{MPa}$, respectively. The Young's modulus of PA-Col and PA-Col/NAC
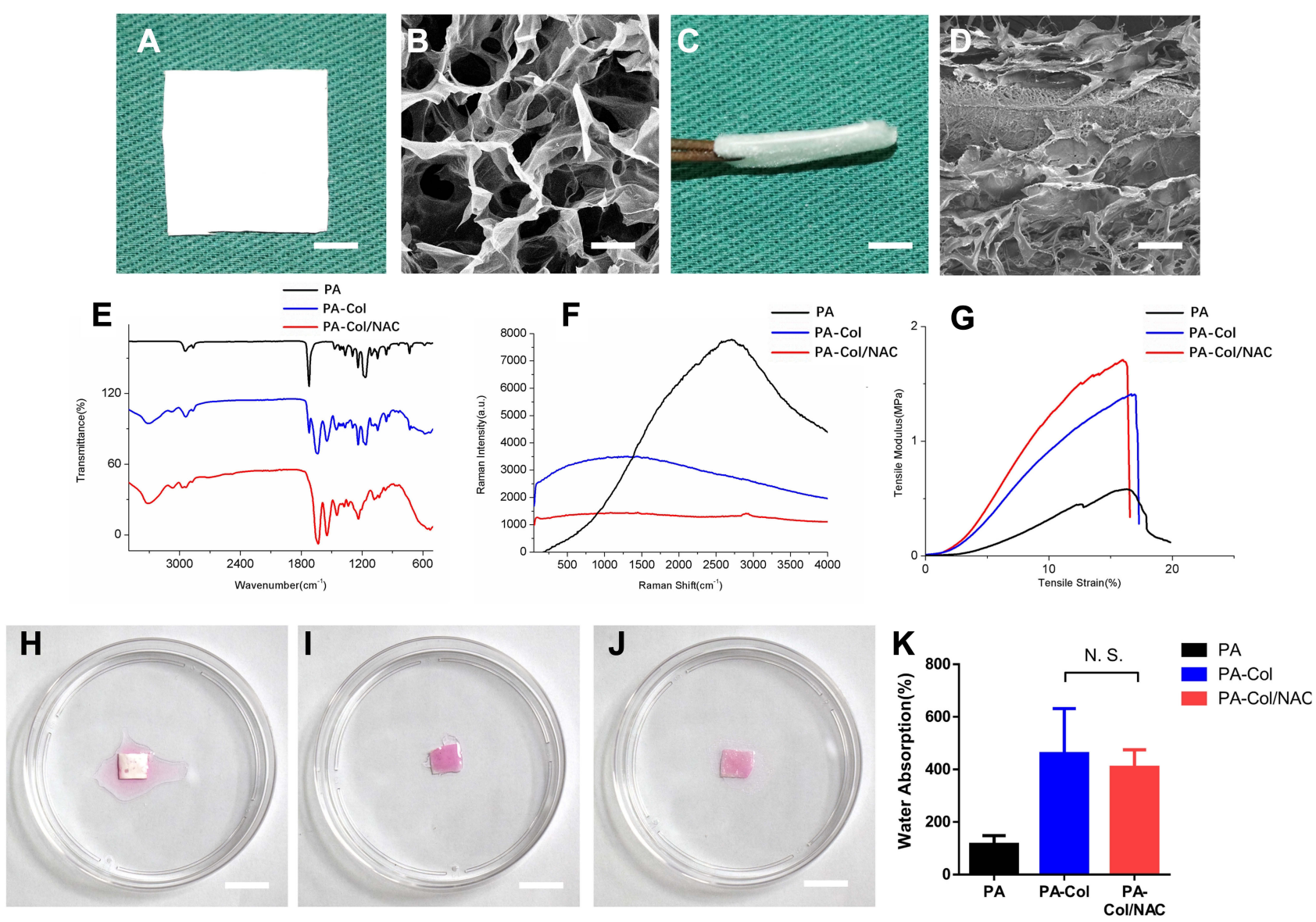

Figure 2 (A and C) Macro photograph of PA-Col/NAC scaffolds from vertical view and from the side view (scale bar I cm). (B and D) Magnified view of A and C (scale bar $100 \mathrm{~nm}$ ). (E) ATR-FTIR spectroscopy, (F) Raman spectroscopy and (G) tensile stress-tensile modulus curve of the scaffolds was displayed. (H-J) Water absorption of three scaffolds indicated by stained normal saline (scale bar $\mathrm{I} \mathrm{cm})$. (K) Water absorption histogram of three scaffolds.

Abbreviation: N.S., not significant. 
scaffold was lower than that of PA scaffold, indicating that PA-Col/NAC and PA-Col scaffolds were much softer than the PA scaffold and hence more suitable for wound dressing.

Water absorption capacity is one of the important factors affecting drug release. ${ }^{24}$ Besides, it is important that wound dressings possess the ability to absorb blood, necrotic tissues and exudate, which is beneficial to the prevention of infection. Figure $2 \mathrm{H}-\mathrm{J}$ shows the condition at which the scaffolds absorbed a liquid similar to the body fluid. According to this results, PA-Col and PA-Col/NAC scaffolds performed better and had higher water absorption capacity than the PA nanofibers. To further examine this property of the scaffolds, Figure $2 \mathrm{~K}$ was plotted to reveal the water absorption capacity of each scaffold. This plot indicated that the water absorption of PA-Col and PA-Col/NAC was superior to that of PA nanofibers, and this was ascribed to the high water absorption capacity of collagen. However, there was no significant difference between PA-Col and PA-Col/NAC scaffold, implying that the NAC or the process of crosslinking did not influence the water absorption property of the scaffolds.

\section{The Cell Biocompatibility and Cell Migration of the PA-Col/NAC Scaffolds in vitro}

In this study, NIH-3T3 cell types were selected for testing the effects of the scaffolds on wound healing in vitro, as fibroblasts were the mainly cell types involving in process of wound healing. ${ }^{32}$ The biocompatibility of each group was inferred from the results of FDA/PI staining and CCK8 assay. Figure 3A shows that there was no significant difference among the three groups on day 1 and 3 . However, on the 7th day, the density of the live cells (marked in green) on the PA-Col and PA-Col/NAC samples was much higher than that of the PA group. This could be due to good cell biocompatibility or water absorption property of collagen. The number of live cells in PA-Col/NAC group was higher than that in PA-Col group whereas the number of dead cells (marked in red) in PA-Col/NAC group was higher than that in PA-Col group, and this was associated with the effects of NAC.

The results of CCK8 assays were also consistent with those indicated in Figure 3A. Figure 3B shows that there was no significant difference among the three groups in the $1 \mathrm{st}$ and 3rd day. However, on the 7th day, the absorbance of PA$\mathrm{Col}$ and $\mathrm{PA}-\mathrm{Col} / \mathrm{NAC}$ at $450 \mathrm{~nm}$ was higher than that of PA, whereas there was no significant difference between PA-Col and PA-Col/NAC. This indicated that NAC had no detectable effect on cell proliferation, and it did not induce cell toxicity.

The general cell migration trend for the three groups is shown in Figure 4A. The closure area was calculated using the Image-Pro software and results are shown in Figure 4B. The analysis revealed that the percent of wound closure area of the PA-Col/NAC group $(65.93 \pm 3.91 \%$ at the 16 th hour and 87.18 $\pm 2.98 \%$ at the 24 th hour) was higher than that of PA-Col group $(61.47 \% \pm 11.29 \%$ at the 16 th hour and $69.27 \% \pm 8.87 \%$ at the
A PA

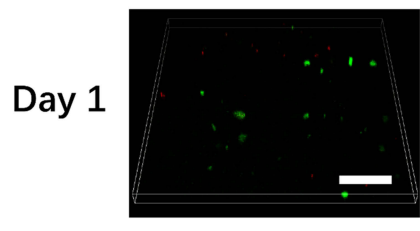

Day 3

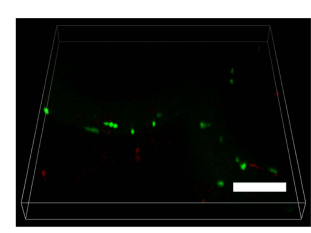

Day 7

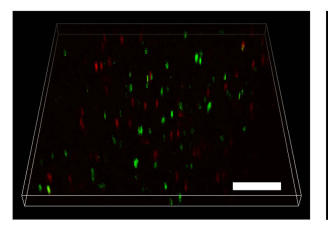

PA-Col
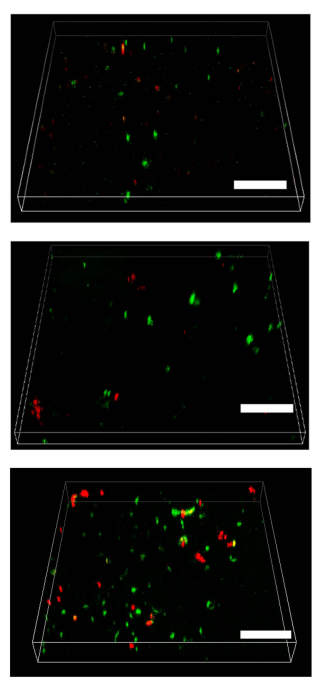

PA-Col/NAC
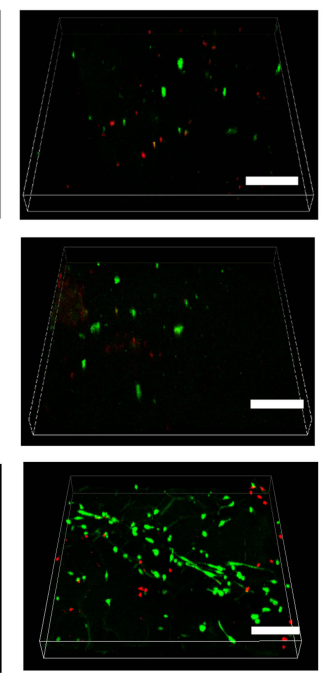

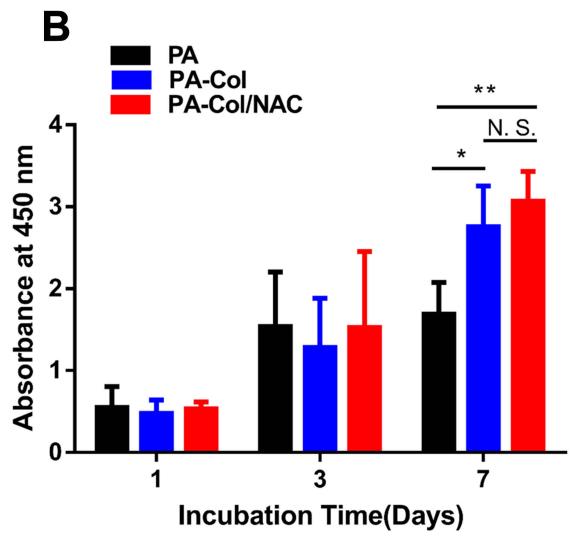

Figure 3 (A) Confocal fluorescent images for FDA/PI staining of NIH 3T3 fibroblasts cultured on the scaffolds for I, 3 and 7 days (scale bars $200 \mu$ m). (B) The CCK8 assay of NIH 3 T3 fibroblasts seeded onto the scaffolds (mean $\pm \mathrm{SD}$; $* \mathrm{P}<0.05, * * \mathrm{P}<0.0 \mathrm{I}$ ).

Abbreviation: N.S., not significant. 

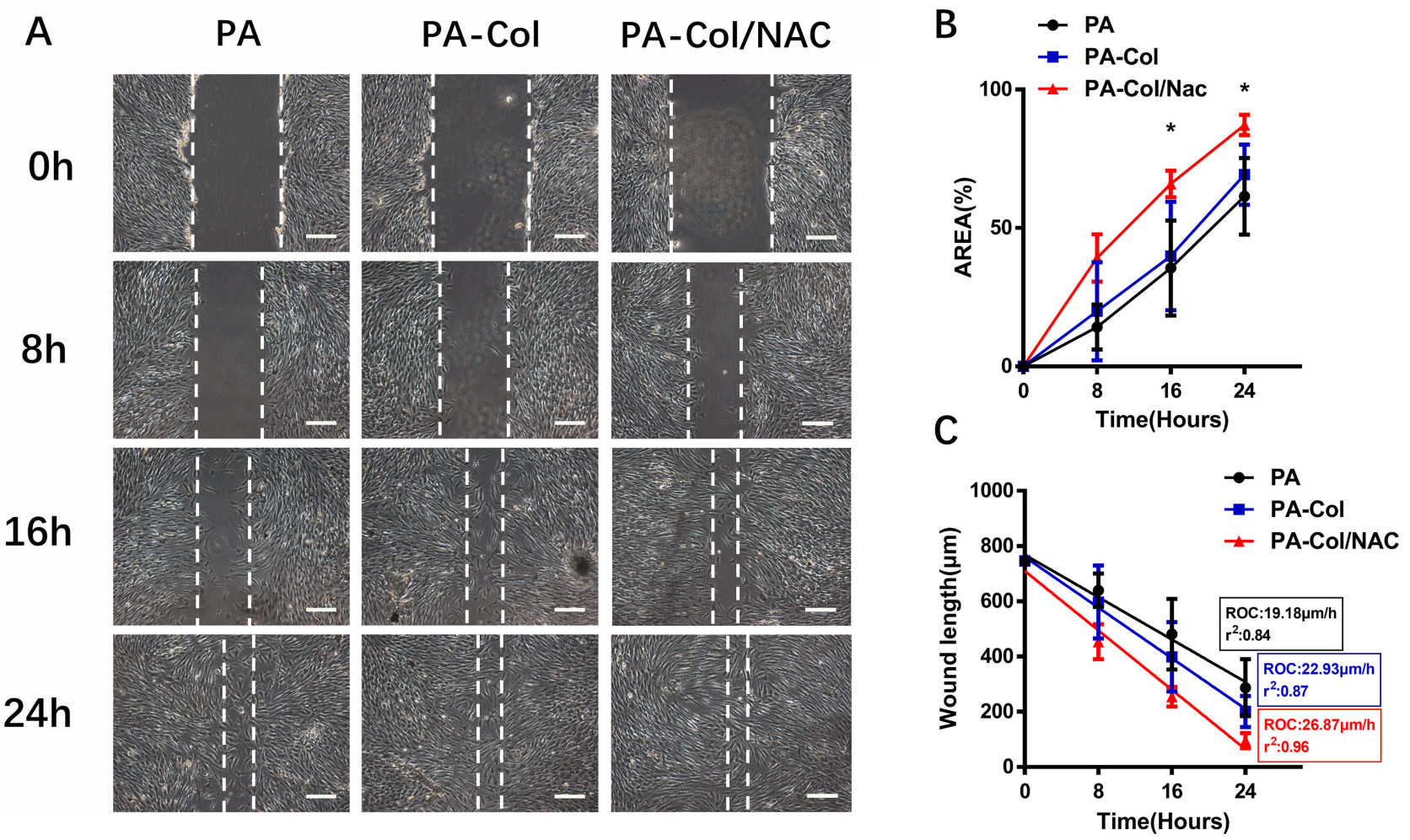

Figure 4 (A) Images from scratch experiment for three groups at different time point (scale bars $200 \mu \mathrm{m})$. (B) Closure area (\%) of the scaffolds at different time points $\left(* \mathrm{P}<0.05\right.$, PA-Col vs PA-Col/NAC). (C) A scatter plot was used to display the width of the wound over time and the rate of closure (ROC) was calculated. To generate the $r^{2}$ value, a linear regression was run on the graph using the GraphPad Prism software.

24th hour) and PA group $(35.53 \% \pm 13.99 \%$ at the 16th hour and $39.86 \% \pm 15.97 \%$ at the 24 th hour). To further examine the wound closure rate, the wound width-time curve (Figure 4C) was plotted. Linear regression was used to examine the average rate of wound healing and calculate the rate of closure. The rate of closure of PA-Col/NAC group $26.87 \mu \mathrm{m} / \mathrm{h}(\mathrm{r} 2=0.96)$ was higher than that of PA group $19.18 \mu \mathrm{m} / \mathrm{h}(\mathrm{r} 2=0.84)$ and PA-Col group $22.93 \mu \mathrm{m} / \mathrm{h}\left(\mathrm{r}^{2}=0.87\right)$.

\section{Sustained Release of PA-COL/NAC Scaffolds}

The drug release profiles of the scaffolds are as shown in Figure 5 and the process of sustained release was divided into two parts. For the first $24 \mathrm{hrs}$, we could infer that most of the NAC were released for the two scaffolds and the cumulative release percentage of PA-Col/NAC $(54.53 \%$ $\pm 7.45 \%)$ was higher than that of Col-NAC $(35.75 \%$ $\pm 0.84 \%$ ) in Figure 5B. There was a sustained drug release from day 7 to day 14. Moreover, the cumulative drug released from PA-Col/NAC $(58.56 \% \pm 8.77 \%)$ was higher than that of Col-NAC $(40.41 \% \pm 0.49 \%)$. This indicated that the PA-Col/NAC scaffolds displayed a good drug sustained release profile.

\section{In vivo Evaluation of the Therapeutic Effects of PA-Col/NAC on Rat Wound Model}

The wound model producer fabricated by $3 \mathrm{D}$ printing method was used to create three oval (major axis $=2 \mathrm{~cm}$, minor axis $=1 \mathrm{~cm}$ ) full-thickness wounds on the dorsa of the rat. These were then covered with the three types of scaffolds. After implantation, the dorsal area was covered with an air-permeable film to prevent the scaffolds from falling from the wound. The macro views of the wound at different time-points are shown in Figure 6A. To further analyze the percentage of wound area, the curve shown in Figure 6B was plotted. The percentage wound area for the three scaffolds before day 3 was almost similar. However, the percent of wound closure for PA-Col/NAC $(69.3 \%$ $\pm 11.47 \%)$ was higher than that of PA $(37.95 \% \pm 10.36 \%)$ and PA-Col $(54.18 \% \pm 12.51 \%)$ on day 7 . On day 14 , all the rats in $\mathrm{PA}-\mathrm{Col} / \mathrm{NAC}$ group presented almost complete wound healing $(86.17 \% \pm 5.46 \%)$, while the percentage of 
A

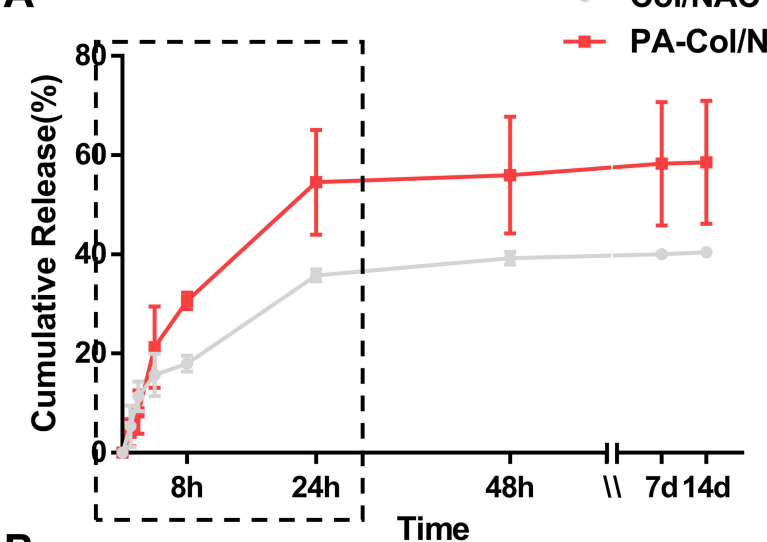

B

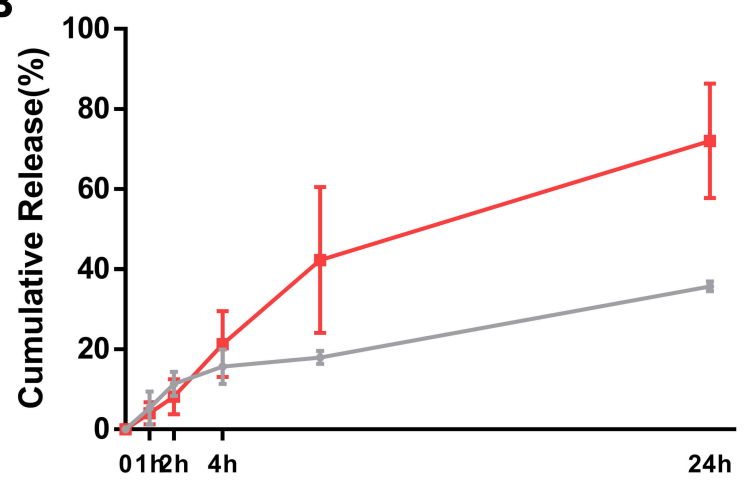

Figure 5 Time-dependent cumulative release profiles of NAC by PA-Col/NAC and Col/NAC.

Notes: (A) Whole time of the cumulative release profile; (B) first 24 hours of the profile. The gray curve demonstrated that NAC has been released up to $35.75 \%$ $\pm 0.84 \%$ in $24 \mathrm{~h}$ and followed by sustained release up to $40.41 \% \pm 0.49 \%$ in 14 th day, while the red curve indicated that NAC has been released up to $54.53 \% \pm 7.45 \%$ in $24 \mathrm{~h}$ and followed by sustained release up to $58.56 \% \pm 8.77 \%$ in 14 th day.

wound closure for PA $(55.15 \% \pm 12.24 \%)$ and PA-Col $(73.5 \% \pm 7.46 \%)$ were much lower.

The results of the histological tests are shown in Figure 7. The unhealed areas in the three groups were distinguished from normal areas by examining the subcutaneous tissues. The sections between the black dash lines showed lighter color with no cutaneous appendages such as hair follicles and sebaceous glands, indicating that the process of wound healing was not complete. The plot shown in Figure $7 \mathrm{M}$ presents the length of the unhealed wound. The wound length for PA-Col/NAC $(1757.3 \mu \mathrm{m} \pm 1185.2 \mu \mathrm{m})$ was shorter than that of PA-Col $(3927.8 \mu \mathrm{m} \pm 1849.5 \mu \mathrm{m})$ and PA $(5223.8 \mu \mathrm{m} \pm$ $2087.7 \mu \mathrm{m})$.

Formation of new epidermis reflected the status of the wound healing process. The HE staining images (Figure 7A-F) revealed that the epidermis of the PA group was discontinuous, whereas the other groups presented complete and continuous epidermis. The thickness of the new epidermis is shown in Figure 7B, D and F. To quantify this measurement, the curve shown in Figure $7 \mathrm{~N}$ was plotted. The thickness of PA-Col/NAC $(67.19 \mu \mathrm{m} \pm 10.60 \mu \mathrm{m})$ was much higher than that of PA-Col $(46.71 \mu \mathrm{m} \pm 13.40 \mu \mathrm{m})$ and PA (38.99 $\mu \mathrm{m} \pm 13.59 \mu \mathrm{m})$.

Collagen regeneration plays an important role in the wound healing process. Figure $7 \mathrm{G}-\mathrm{L}$ shows the Masson staining results of the samples indicating that the new collagen mass in PA and PA-Col at 14 days appears to be irregularly aligned, with high infiltration of inflammatory cells, and high deposition of collagen bundle in PA-Col NAC, uniformly and regularly arranged. This phenomenon indicated that PA-Col/NAC group exhibited better wound healing than PA and PA-Col groups.

To examine the angiogenesis process in the samples, CD31 staining was performed to calculate the number of blood vessels. Figure $8 \mathrm{~A}-\mathrm{F}$ shows the CD31 staining results of the sample. The results shown in Figure $8 \mathrm{G}$ revealed that the number of vessels in PA-Col/NAC group (75.5 \pm 26.1$)$ was higher than that of PA (49.3 $\pm 19.7)$ and PA-Col (48.3 \pm 15.4$)$.

\section{Discussion}

In this study, multi-layered PA-Col/NAC scaffolds were fabricated from PA nanofibers and collagen loaded with NAC. We found that the PA-Col/NAC scaffolds displayed excellent water absorption capacity, porosity, biocompatibility, and sustained drug release profile. Most importantly, the scaffolds promoted wound healing in SD rats.

Polyamide (PA) is a polymer with broad applications in a variety of fields. It is a commonly used biomaterial in medicine due to its good mechanical strength and excellent biocompatibility. ${ }^{33}$ Moreover, compared to poly (lactic acid) and polycaprolactone, PA possess certain advantages such as high mechanical strength, flexibility, rigidity and structural similarity to peptides (due to amide bonds). ${ }^{34}$ Although its biodegradability is not adjustable like other polyesters, it is still effective for wound dressing since this property has little influence on this process. One of the essential applications of PA is in membranes used in medicine and life science such as matrices and scaffolds. ${ }^{35}$ Using the electrospinning technology, PA nanofibers can be manipulated for various applications. ${ }^{36} \mathrm{PA}$ nanofibers have been shown to possess three-dimensional interactive porous network structure and high specific surface area. ${ }^{37}$ This structure is equivalent to ECM in biological tissues, and therefore provide ideal environment for cell adhesion and proliferation. ${ }^{38}$ Besides, the direction and orientation of nanofibers that were feature of circumferetially or axially aligned structures might affect the 
A

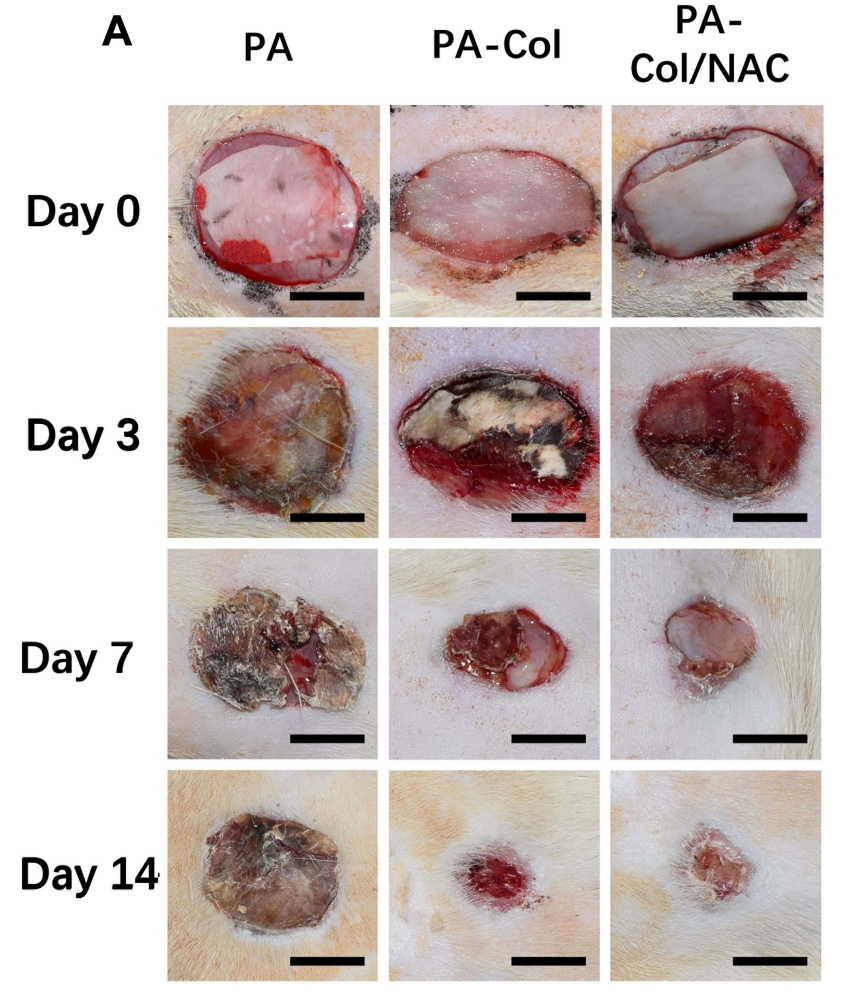

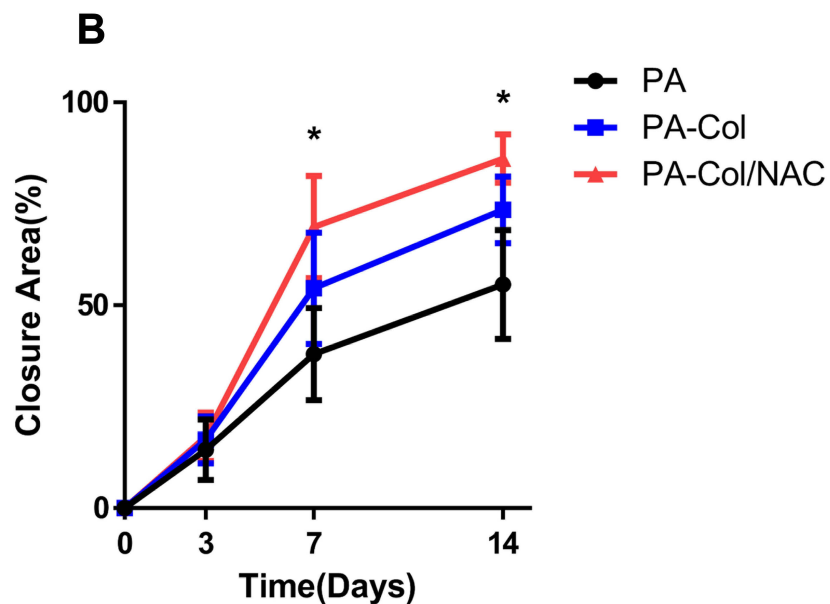

Figure 6 The results of implantation in vivo indicated that PA-Col/NAC group possessed the optimal treatment effects among three groups. Notes: (A) The macro views of three groups treated with the scaffolds (scale bar I cm). (B) Closure area $(\%)$ at different time points after treatment $(\mathrm{mean} \pm \mathrm{SD}$; $* \mathrm{P}<0.05$, PA-Col vs PA-Col/NAC).

vascularization of the scaffolds. ${ }^{39}$ Therefore, in this study, PA was used to prepare PA electrospun nanofibers for fabrication of the PA-Col/NAC scaffold.

Collagen is one of the most common biomaterials used in wound healing. ${ }^{40}$ In this study, collagen was chosen due to the following: i) collagen can achieve sustained drug release via a simple chemical crosslinking method (using the NHS-EDC mixture); ii) given that PA is highly resistant to solvents and body fluids, it might negatively influence the wound healing process. Therefore, incorporating PA to biomaterials such as collagen which have high hygroscopicity remarkably transforms the hydrophobicity of PA. In this study, the water absorption capacity of PACol and PA-Col/NAC scaffolds were similar and higher than that of PA. iii) as the material to directly interact with the wound surface, the collagen mimics the extracellular matrix and provides favorable conditions for cell adhesion and proliferation.

NAC was used to promote wound healing in this study. ${ }^{11}$ As a ROS scavenger, NAC regulates excessive inflammatory response and prevents oxidative stress triggered by ROS. ${ }^{41}$ Feng et al leveraged on the ROS scavenging properties of
NAC and anti-apoptosis effects to promote bone generation. ${ }^{42}$ Deniz et al demonstrated that NAC is a common potent antioxidant which prevents necrosis of stasis when applied topically. ${ }^{43}$ Tsai et al demonstrated that NAC promotes cell proliferation, cell migration, wound healing and collagen expression via the MMP-1 via PKC/Stat3 signaling pathway. ${ }^{9}$

NAC has been shown to accelerate the wound healing process via promotion of the epidermal maturity and angiogenesis. Gill et al illustrated that MMP-1 is important for the reconstruction of the basement membrane and the proliferation of epidermal cells and actin cytoskeletal reorganization all of which participate in for the regeneration of the epidermis. ${ }^{44}$ In this study, we could observed that PA-Col/NAC group achieved the best repairing effects of the epidermis among the three groups. Albasanz-Puig et al reported that NAC activates Stat3 to regulate vascular endothelial growth factor (VEGF) and facilitate the growth of new vessels. ${ }^{45}$ This is in accordance with the results of our study in which the number of CD31 positive vessels in PA-Col/NAC group was higher than that in PA and PA-Col group.

We successfully fabricated scaffolds with multi-layered structure. This method has been widely used in the 


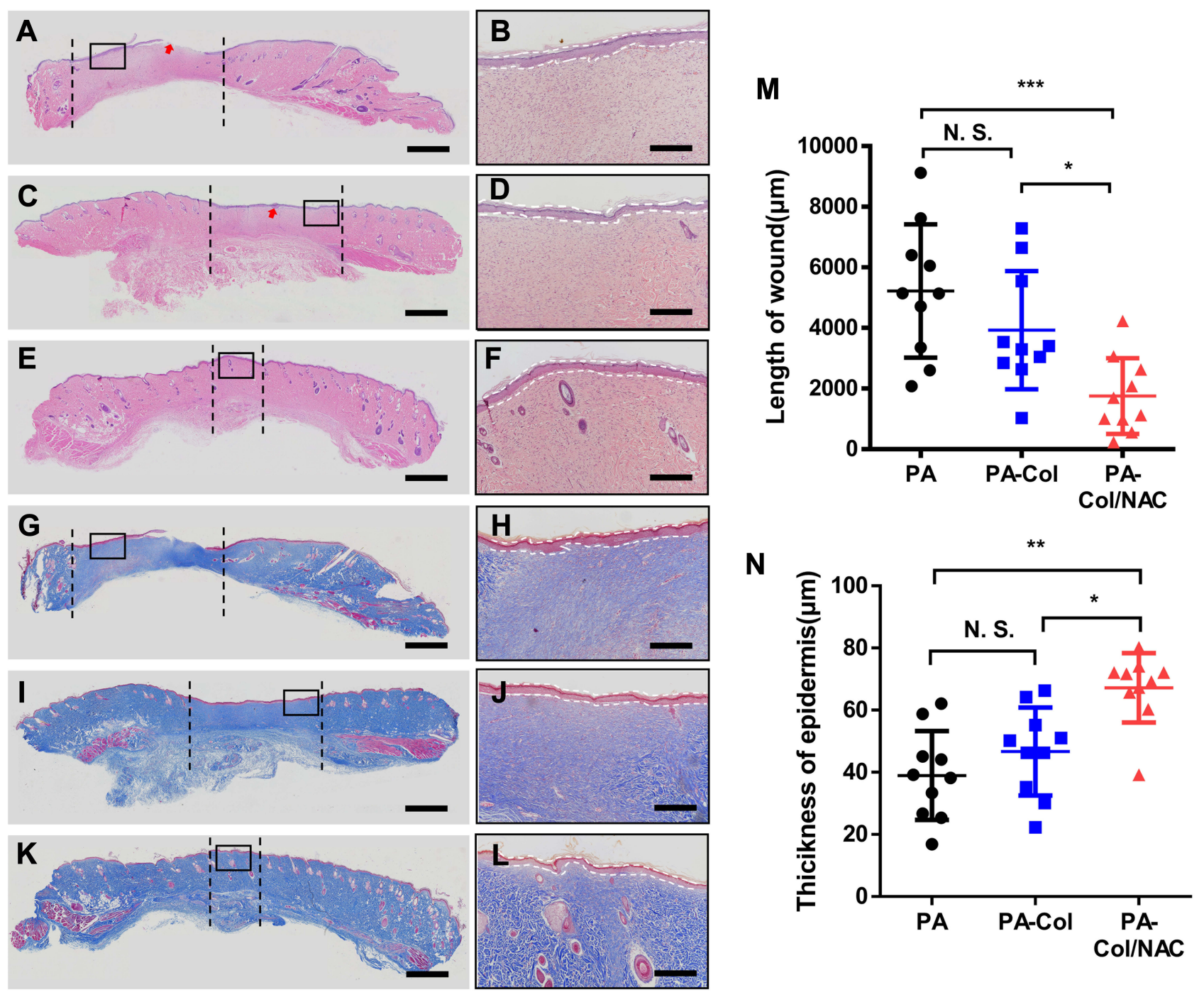

Figure 7 Histological analysis revealed that the PA-Col/NAC group reached better repair effect at day 14.

Notes: (A-F) H\&E staining and (G-L) Masson staining of wound section after 14 days treatment. Red arrows: remaining scaffolds. The section between the black dash lines: unhealed area. Scale bars: $1000 \mu \mathrm{m}$. Magnified view of the region in (A, C, E, G, I, and K) shows thickness of epidermal layer indicated by white dash lines. Scale bars: $400 \mu \mathrm{m}$. (A, B, $\mathbf{G}$ and $\mathbf{H})$ PA group. (C, D, I and J) PA-Col group. (E, F, K and L) PA-Col/NAC group. Analysis of (M) length of wound and (N) thickness of epidermis is shown. Mean \pm SD; *P<0.05; $* * \mathrm{P}<0.01 ; * * * \mathrm{P}<0.001$.

Abbreviation: N.S., not significant.

fabrication of composites. Zhang et al prepared sandwichlike fibers/sponge composites using a similar method to prevent the recurrence of tumor and metastasis and combined it with chemotherapy and hemostasis. The scaffold carrying the gelation loaded with cisplatin produced good hemostasis and chemotherapy effects. In this study, the multi-layered structure also played an important role in the fabricated PA-Col /NAC scaffolds. The PA nanofibers enhanced the stability of the scaffolds at the wound area. The collagen loaded with NAC on each side had direct contact with the wound. Collagen provided a favorable environment for wound healing and controlled the sustained release of the NAC to the wound. NAC promoted the wound healing process by resisting ROS, accelerating the maturation of the epidermis and facilitating angiogenesis.

\section{Conclusion}

In conclusion, this study successfully fabricated a multilayered scaffold for would dressing. The scaffold consisted of PA nanofibers and collagen loaded with NAC, which promoted the wound healing process. This novel PA-Col/NAC scaffold displayed excellent water absorption capacity, mechanical properties, biocompatibility, and ability to steadily release NAC. These properties 

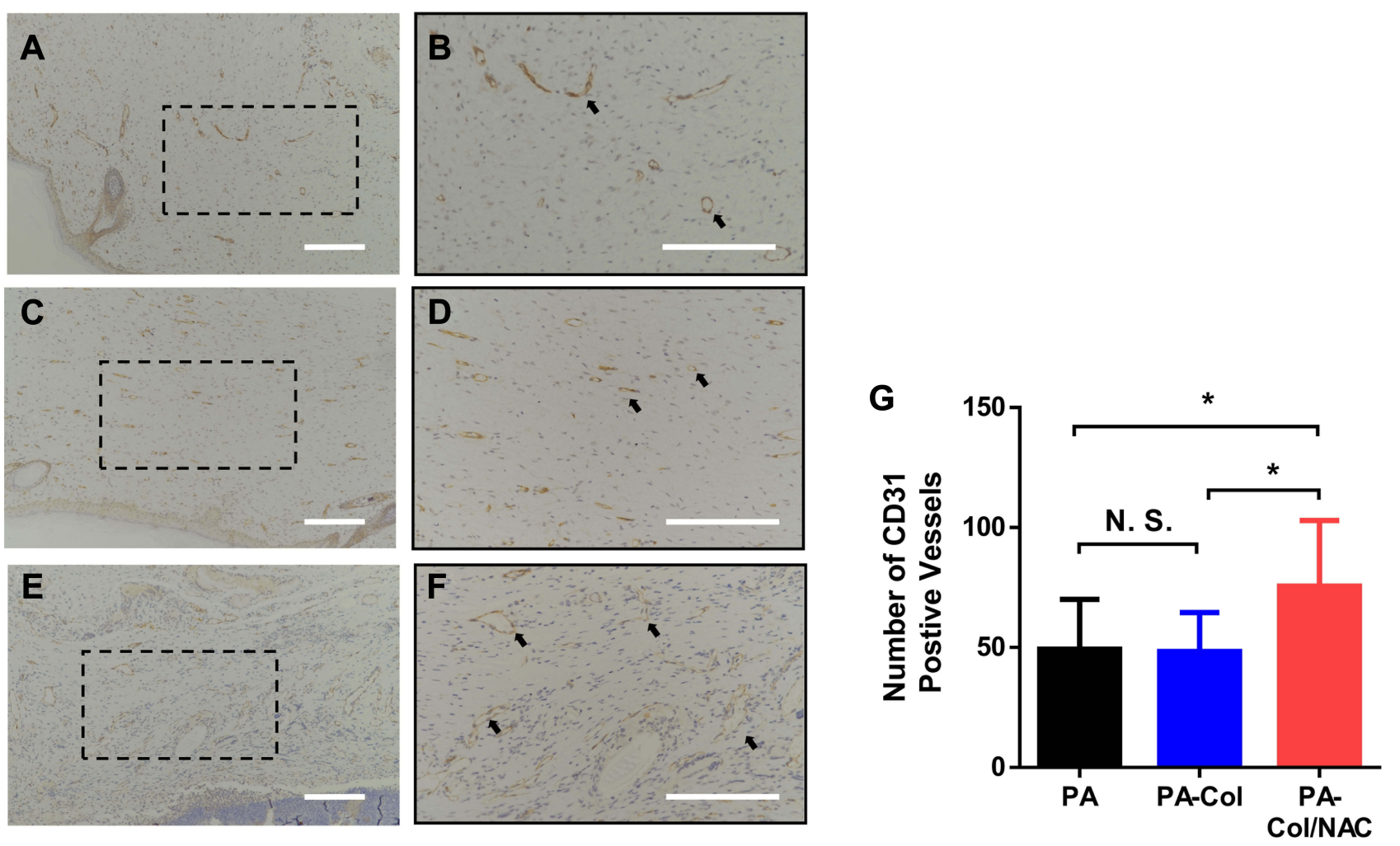

Figure 8 Immunohistochemical staining for CD3I showed significant increase of number of vessels in PA-Col/NAC group compared with the other groups. Notes: (A-F) Vascular endothelial cells that showed CD3I antibody positive were marked by black arrows. Scale bars: $200 \mu \mathrm{m}$. (A and B) PA group. (C and D) PA-Col group. (E and F) PA-Col/NAC group. (G) Analysis of number of CD3I positive vessels on skin tissue sections. $* \mathrm{P}<0.05$. Abbreviation: N.S., not significant.

promoted cell proliferation and migration in vitro. The scaffold also enhanced the repair of an oval rat wound indicating a promising application in wound healing.

\section{Acknowledgments}

This work was supported by the National Key R\&D Program of China (2019YFA0110500), and the National Natural Science Foundation of China (No. 81701922 and 81873941). The authors thank the Analytical \& Testing Center of HUST and the assistance of Doctor Zaipeng Liu.

\section{Disclosure}

The authors report no conflicts of interest in this work.

\section{References}

1. Sen CK, Gordillo GM, Roy S, et al. Human skin wounds: a major and snowballing threat to public health and the economy. Wound Repair Regen. 2009;17(6):763-771. doi:10.1111/wrr.2009.17.issue-6

2. Han G, Ceilley R. Chronic wound healing: a review of current management and treatments. Adv Ther. 2017;34(3):599-6103. doi:10.1007/ s12325-017-0478-y

3. Moura D, Souza MT, Liverani L, et al. Development of a bioactive glass-polymer composite for wound healing applications. Mater Sci Eng C. 2017;76:224-232. doi:10.1016/j.msec.2017.03.037
4. Rahmani Del Bakhshayesh A, Annabi N, Khalilov R, et al. Recent advances on biomedical applications of scaffolds in wound healing and dermal tissue engineering. Artif Cells Nanomed Biotechnol. 2018;46(4):691-705. doi:10.1080/21691401.2017.1349778

5. Samuni Y, Goldstein S, Dean OM, Berk M. The chemistry and biological activities of N-acetylcysteine. Biochim Biophys Acta. 2013;1830:4117-4129. doi:10.1016/j.bbagen.2013.04.016

6. Yanping P, Huan L, Yi Y, et al. Biological activities and potential oral applications of $\mathrm{N}$-acetylcysteine: progress and prospects. Oxid Med Cell Longev. 2018;2018:1-14.

7. Dunnill C, Patton T, Brennan J, et al. Reactive oxygen species (ROS) and wound healing: the functional role of ROS and emerging ROS-modulating technologies for augmentation of the healing process. Int Wound J. 2017;14(1):89-96. doi:10.1111/iwj.2017.14.issue-1

8. Wei X, Song H, Semenkovich CF. Insulin-regulated protein palmitoylation impacts endothelial cell function. Arterioscler Thromb Vasc Biol. 2014;34(2):346-3548. doi:10.1161/ATVBAHA.113.302848

9. Tsai ML, Huang HP, Hsu JD, et al. Topical N-acetylcysteine accelerates wound healing in vitro and in vivo via the $\mathrm{PKC} / \mathrm{Stat} 3$ pathway. Int J Mol Sci. 2014;15(5):7563-7578. doi:10.3390/ijms15057563

10. Manniello MD, Del Gaudio P, Aquino RP, Russo P. Clarithromycin and $\mathrm{N}$-acetylcysteine co-spray-dried powders for pulmonary drug delivery: a focus on drug solubility. Int J Pharm. 2017;533(2):463469.13. doi:10.1016/j.ijpharm.2017.03.079

11. Oguz A, Uslukaya O, Alabalık U, Turkoglu A, Kapan M, Bozdag Z. Topical $\mathrm{N}$-acetylcysteine improves wound healing comparable to dexpanthenol: an experimental study. Int Surg. 2015;100(4):656-661. doi:10.9738/INTSURG-D-14-00227.1

12. Sanguinetti CM. N-acetylcysteine in COPD: why, how, and when? Multidiscip Respir Med. 2015;11. doi:10.1186/s40248-016-0039-2 
13. Gilmartin DJ, Soon A, Thrasivoulou C, Phillips AR, Jayasinghe SN, Becker DL. Sustained release of $\mathrm{Cx} 43$ antisense oligodeoxynucleotides from coated collagen scaffolds promotes wound healing. Adv Healthc Mater. 2016;5(14):1786-1799. doi:10.1002/adhm.201600175

14. Aurora A, Beasy A, Rizzo JA, Chung KK. The use of a silver-nylon dressing during evacuation of military burn casualties. $J$ Burn Care Res. 2018;39(4):593-597. doi:10.1093/jbcr/irx026

15. Liu X, Dan N, Dan W. Insight into the collagen assembly in the presence of lysine and glutamic acid: an in vitro study. Mater Sci Eng CMater Biol Appl. 2017;70(Pt 1):689-700. doi:10.1016/j.msec.2016.09.037

16. Parenteau-Bareil R, Gauvin R, Cliche S, Gariépy C, Germain L, Berthod F. Comparative study of bovine, porcine and avian collagens for the production of a tissue engineered dermis. Acta Biomater. 2011;7(10):3757-3765. doi:10.1016/j.actbio.2011.06.020

17. Yu SM, Li Y, Kim D. Collagen mimetic peptides: progress towards functional applications. Soft Matter. 2011;7(18):7927-7938. doi: $10.1039 / \mathrm{c} 1 \mathrm{sm} 05329 \mathrm{a}$

18. Li Y, Yu SM. Targeting and mimicking collagens via triple helical peptide assembly. Curr Opin Chem Biol. 2013;17(6):968-975. doi:10.1016/j.cbpa.2013.10.018

19. Ogino S, Morimoto N, Sakamoto M, et al. Efficacy of the dual controlled release of HGF and bFGF impregnated with a collagen/gelatin scaffold. J Surg Res. 2018;221:173-182. doi:10.1016/j.jss.2017.08.051

20. Chattopadhyay S, Raines RT, Glick GD. Review collagen-based biomaterials for wound healing. Biopolymers. 2014;101(8):821-833. doi:10.1002/bip.22486

21. Tajirian AL, Goldberg DJ. A review of sutures and other skin closure materials. J Cosmet Laser Ther. 2010;12(6):296-302. doi:10.3109/ 14764172.2010 .538413 .

22. Dias FTG, Ingracio AR, Nicoletti NF, et al. Soybean-modified polyamide- 6 mats as a long-term cutaneous wound covering. Mater Sci Eng C Mater Biol Appl. 2019;99:957-968. doi:10.1016/j. msec.2019.02.019

23. Hosseinzadeh S, Soleimani M, Vossoughi M, et al. Study of epithelial differentiation and protein expression of keratinocyte-mesenchyme stem cell co-cultivation on electrospun nylon/B. vulgaris, extract composite scaffold. Mater Sci Eng C. 2017;75:653-662. doi:10.1016/j. msec.2017.02.101

24. Motealleh B, Zahedi P, Rezaeian I, Moghimi M, Abdolghaffari AH, Zarandi MA. Morphology, drug release, antibacterial, cell proliferation, and histology studies of chamomile-loaded wound dressing mats based on electrospun nanofibrous poly( $\varepsilon$-caprolactone)/polystyrene blends. J Biomed Mater Res B Appl Biomater. 2014;102:977-987. doi:10.1002/jbm.b.v102.5

25. Lu MM, Wang QJ, Chang ZM, et al. Synergistic bactericidal activity of chlorhexidine-loaded, silver-decorated mesoporous silica nanoparticles. Int J Nanomedicine. 2017;12:3577-3589. doi:10.2147/IJN

26. Hou J, Chen L, Liu Z, et al. Sustained release of N-acetylcysteine by sandwich structured polycaprolactone/collagen scaffolds for wound healing. J Biomed Mater Res A. 2019;107(7):1414-1424. doi:10.1002/ jbm.a.v107.7

27. Yan $\mathrm{Z}$, Wang $\mathrm{W}, \mathrm{Wu} \mathrm{Y}$, et al. Zinc oxide nanoparticle-induced atherosclerotic alterations in vitro and in vivo. Int J Nanomedicine. 2017;12:4433-4442. doi:10.2147/IJN.S134897

28. Fan L, Zhang Q, Yang Z, et al. Improving permeation and antifouling performance of polyamide nanofiltration membranes through the incorporation of arginine. ACS Appl Mater Interfaces. 2017;9 (15):13577-13586. doi:10.1021/acsami.7b00159

29. Hu Z, Jie Z, Taofen W, et al. In-situ preparation of silver salts/ collagen fiber hybrid composites and their photocatalytic and antibacterial activities. J Hazard Mater. 2018;359:274-280. doi:10.1016/ j.jhazmat.2018.07.043
30. Benjakul S, Thiansilakul Y, Visessanguan W, et al. Extraction and characterisation of pepsin-solubilised collagens from the skin of bigeye snapper (Priacanthus tayenus and Priacanthus macracanthus). J Sci Food Agric. 2010;90(1):132-138. doi:10.1002/ jsfa.3795

31. Zhang Y, Yin LH, Feng F. Study on Fr-Raman scattering of cystine, cysteine and acetylcysteine. Chin Pharm Affairs. 2010; 24:447-449.

32. Rong X, Chu W, Zhang H, et al. Antler stem cell-conditioned medium stimulates regenerative wound healing in rats. Stem Cell Res Ther. 2019;10(1):326. doi:10.1186/s13287-019-1457-9

33. Yao MZ, Huang-Fu MY, Liu HN, Wang XR, Sheng X, Gao JQ. Fabrication and characterization of drug-loaded nano-hydroxyapatite/polyamide 66 scaffolds modified with carbon nanotubes and silk fibroin. Int $J$ Nanomedicine. 2016;11:6181-6194 . doi:10.2147/IJN.S106929

34. Winnacker M. Polyamides and their functionalization: recent concepts for their applications as biomaterials. Biomater Sci. 2017;10 (1039):C7BM00160F.

35. Sundaram S, H G J, Martin JM. Pall corporation advocates the "broadscale substitution". PDA J Pharm Sci Technol. 2002;56(1):2-3.

36. J S S, D B C, Rabolt JF. Effect of the electrospinning process on polymer crystallization chain conformation in Nylon6 and Nylon12. Macromolecules. 2004;37(3):877-881. doi:10.1021/ ma0351569

37. Liu M, Duan XP, Li YM, Yang DP, Long YZ. Electrospun nanofibers for wound healing. Mater Sci Eng C Mater Biol Appl. 2017;1 (76):1413-1423. doi:10.1016/j.msec.2017.03.034

38. Garcia-Orue I, Gainza G, Gutierrez FB, et al. Novel nanofibrous dressings containing rhEGF and Aloe vera for wound healing applications. Int $J$ Pharm. 2017;523(2):556-566. doi:10.1016/j. ijpharm.2016.11.006

39. Niu Z, Wang X, Meng X, et al. Controllable fiber orientation and nonlinear elasticity of electrospun nanofibrous small diameter tubular scaffolds for vascular tissue engineering. Biomed Mater. 2019;14 (3):035006. doi:10.1088/1748-605X/ab07f1

40. Zhou T, Sui B, Mo X, Sun J. Multifunctional and biomimetic fish collagen/bioactive glass nanofibers: fabrication, antibacterial activity and inducing skin regeneration in vitro and in vivo. Int $J$ Nanomedicine. 2017;12:3495-3507. doi:10.2147/IJN

41. Shen Y, Gong S, Li J, et al. Co-loading antioxidant N-acetylcysteine attenuates cytotoxicity of iron oxide nanoparticles in hypoxia/reoxygenation cardiomyocytes. Int J Nanomedicine. 2019;14:6103-6115. doi:10.2147/IJN.S209820

42. Feng Y, Wang L, Ma X, et al. Effect of reactive oxygen species overproduction on osteogenesis of porous titanium implant in the present of diabetes mellitus. Biomaterials. 2013;34(9):2234-2243. doi:10.1016/j.biomaterials.2012.12.023

43. Deniz M, Borman H, Seyhan T, Haberal M. An effective antioxidant drug on prevention of the necrosis of zone of stasis: $\mathrm{N}$-acetylcysteine. Burns. 2013;39(2):320-325. doi:10.1016/j. burns.2012.06.015

44. Gill SE, Parks WC. Metalloproteinases and their inhibitors: regulators of wound healing. Int $J$ Biochem Cell Biol. 2008;40 (6):1334-1347. doi:10.1016/j.biocel.2007.10.024

45. Albasanz-Puig A, Murray J, Namekata M, et al. Opposing roles of STAT-1 and STAT-3 in regulating vascular endothelial growth factor expression in vascular smooth muscle cells. Biochem Biophys Res Commun. 2012;428(1):179-184. doi:10.1016/j.bbrc. 2012.10.037 


\section{Publish your work in this journal}

The International Journal of Nanomedicine is an international, peerreviewed journal focusing on the application of nanotechnology in diagnostics, therapeutics, and drug delivery systems throughout the biomedical field. This journal is indexed on PubMed Central, MedLine, CAS, SciSearch ${ }^{\mathbb{B}}$, Current Contents ${ }^{\mathbb{B}} /$ Clinical Medicine,
Journal Citation Reports/Science Edition, EMBase, Scopus and the Elsevier Bibliographic databases. The manuscript management system is completely online and includes a very quick and fair peer-review system, which is all easy to use. Visit http://www.dovepress.com/ testimonials.php to read real quotes from published authors. 